\title{
DERMATITIS ARTEFACTA AND SEXUAL ABUSE
}

\author{
MADHULIKA A. GUPTA, M.D., F.R.C.P.C. AND ADITYA K. GUPTA, M.D., F.R.C.P.C.
}

Case 1: This 16-year-old woman was referred for psychiatric management of her dermatitis artefacta that presented as superficial linear abrasions on her thighs and arms. During psychotherapy, the patient disclosed that her father had started sexually molesting her when she was about 8 years old. By age 10 years her father was having sexual intercourse with her. Initially, the patient confused her father's sexual advances with affection, but by age 10 years she became acutely aware of the abusive nature of their relationship. At this time, the father threatened to leave the family if the patient disclosed the sexual abuse. This was very threatening for the patient, since it would mean that her mother and her siblings would end up on welfare. The father then blamed the patient for "seducing him" and continued to abuse her sexually; the patient felt guilty and "bad."

The patient started making superficial linear abrasions on the anterior aspects of her thighs. She described that it helped her "release tension" because the pain helped her to cope with her guilt. The patient was essentially "punishing herself" by inflicting the lesions on herself. The patient also felt "very fat and ugly" and the scars on her body helped to validate this. During the course of therapy, the patient had much difficulty coping with the unresolved anger that began to surface as she acknowledged her feelings regarding the sexual abuse. Initially, she could cope with her anger only by cutting herself, and the symptoms of her dermatitis artefacta became worse. After about 1 year of psychotherapy she was able to acknowledge her feelings more effectively and became much more assertive. This was associated with a marked decrease in the frequency of her self-mutilative behavior.

Case 2: This 29-year-old woman was referred for psychiatric management of her dermatitis artefacta. The patient would typically present to her family doctor with rectangular abrasions on her forearms. The self-inflicted nature of the lesions was obvious on inspection, but the patient denied remembering that she caused the injury. The patient entered psychotherapy once weekly, and after approximately 6 months, she began to have flashbacks about being sexually abused regularly by an older brother when she was be-

From the Department of Psychiatry, University of Michigan Medical School, Ann Arbor, Michigan, and Division of Dermatology, Department of Medicine, University of Toronto, Faculty of Medicine, Toronto, Ontario, Canada.

Address for correspondence: M.A. Gupta, M.D., Box 0704, CFOB, Department of Psychiatry, University of Michigan Medical School, 1500 East Medical Center Drive, Ann Arbor, MI 48109-0704. tween ages $5-8$ years. The patient described dissociative experiences when she was being subjected to the abuse. For example, she felt like "she was watching someone else being abused" and remembers "not feeling anything" while her brother was raping her. Over several months she was able to recognize that she would dissociate whenever faced with emotionally disturbing situations, especially those involving anger. During these dissociative states she would inflict the rectangular abrasions on herself. The patient experienced a significant improvement in the frequency of her dissociative experiences and her self-mutilative behavior as she acknowledged her anger toward her brother more directly. Chlorpromazine, $25 \mathrm{mg}$ qid, prn, also proved to be a valuable adjunct in her therapy, as it helped her to cope with her very strong anger, and decreased the frequency of her dissociative reactions.

\section{DISCUSSION}

Dermatitis artefacta is a term used to describe cutaneous lesions with wide-ranging morphologic features that are wholly self-inflicted. ${ }^{1}$ The psychopathologic syndromes that have been associated with dermatitis artefacta are diverse, ranging from psychosis, mental retardation, Münchausen's syndrome, an immature personality and malingering. ${ }^{1-3}$ While sexual abuse can lead to a wide range of psychopathological disturbances, its association with dermatitis artefacta has not been adequately emphasized in the literature. ${ }^{1}$

We present two patients with dermatitis artefacta, both manifesting as superficial abrasions of the skin. In both patients the skin lesions were associated with a past and/or current history of severe sexual abuse. $\mathrm{Pa}$ tient 1 inflicted the lesions on herself to cope with her unfounded guilt by punishing herself. Body image problems are often a sequelae of childhood sexual abuse, ${ }^{4}$ and a prospective study of patients with dermatitis artefacta showed that $5 \%$ of these patients develop anorexia nervosa, ${ }^{5}$ a syndrome associated with severe body image problems. It is possible that sexual abuse was a common precipitating factor for both the dermatitis artefacta and the body image disorder among these patients. Patient 2 self-inflicted her artifactual lesions during dissociative episodes. The essential feature of dissociative disorders is a disturbance or alteration in the normally integrative functions of identity, memory, or consciousness. ${ }^{6}$ In our patient, there was primarily a disturbance of memory and her symptoms were consistent with a diagnosis of psychogenic 
amnesia. ${ }^{6}$ The patient had considerable unresolved anger from her childhood, which caused her to feel emotionally overwhelmed when faced with anger in her life, and this resulted in dissociative episodes. The pain from the self-inflicted lesions would help her to distract herself further from her anger and emotional pain.

\section{CONCLUSIONS}

It is important to consider the possibility of sexual abuse when assessing a patient with dermatitis artefacta. Patients who have experienced sexual abuse are often reluctant to volunteer this information or may not readily recollect the abuse if they were experiencing dissociative episodes as a result of the psychologic trauma. If the clinician, however, maintains an index of suspicion, the dermatologic and emotional sequelae of the sexual abuse may both be treatable.

\section{DRUG NAME}

chlorpromazine: Thorazine

\section{REFERENCES}

1. Gupta MA, Gupta AK, Haberman HF. The self-inflicted dermatoses: a critical review. Gen Hosp Psychiatry 1987; 9:45-52.

2. Lyell A. Cutaneous artifactual disease. J Am Acad Dermatol 1979; 1:391-407.

3. Fabisch W. Psychiatric aspects of dermatitis artefacta. Br J Dermatol 1980; 102:29-34.

4. Palmer RL, Oppenheimer R, Dignon A, et al. Childhood sexual experiences with adults reported by women with eating disorders: an extended series. $\mathrm{Br} \mathrm{J}$ Psychiatry $1990 ; 156: 699-703$.

5. Sneddon I. Self-inflicted injury: a follow-up study of 43 patients. Br Med J 1975; 1:527-530.

6. American Psychiatric Association. Diagnostic and statistical manual of mental disorders. 3rd Ed. Rev. Washington, DC: American Psychiatric Association, 1987:269.

\section{Pseudo-Red-Man Syndrome}

Red-man syndrome is a well-known erythematous reaction involving the upper trunk in patients who have received vancomycin. The flushing may be associated with pruritus hypotension. It appears to be caused by histamine, other mediators, or vancomycin itself. Teicoplanin is a newer glycopeptide antibiotic with an antimicrobial spectrum similar to that of vancomycin, but with a very long half-life. It is not known to be associated with red-man syndrome. Red-man syndrome appeared to develop in the following patient after he received teicoplanin.

The patient was a 65 -year-old man who had undergone quadruple coronaryartery bypass surgery. He was enrolled in a double-blind trial comparing teicoplanin and cefazolin as prophylaxis against postoperative infection. The intraoperative and postoperative course was uncomplicated. On the fifth postoperative day the patient was noted to have diffuse erythema involving both arms. The erythema started about $10 \mathrm{~cm}$ below the shoulders and extended to the wrists. There was no blanching or warmth. There was complete sparing of the area under his wristwatch. The only other medications that he had been receiving were aspirin and an antihistamine for rhinitis.

I initially considered the possibility that he had red-man syndrome, but he had not received vancomycin, so I was quite puzzled. Then his bathrobe caught my eye. The bright red terry-cloth robe was brand-new. The patient had not been compliant with the manufacturer's instructions, and no one had ever washed it in cold water to remove the sizing.

The red dye from the robe had colored the patient's arms, which were still wet from a shower. From Salit IE. Pseudo-red-man syndrome. N Engl J Med 1993; 328:584-585. 
This document is a scanned copy of a printed document. No warranty is given about the accuracy of the copy. Users should refer to the original published version of the material. 